Trinity University

Digital Commons @ Trinity

Human Communication and Theatre Faculty

Research

Human Communication and Theatre

6-2017

\title{
The Relational Reconnection Function of Social Network Sites
}

Artemio Ramirez Jr.

Erin M. Bryant

Trinity University, ebryant@trinity.edu

John Spinda

Follow this and additional works at: https://digitalcommons.trinity.edu/hct_faculty

Part of the Theatre and Performance Studies Commons

\section{Repository Citation}

Ramirez, A., Jr., Sumner, E. M., \& Spinda, J. (2017). The relational reconnection function of social network sites. New Media \& Society, 19(6), 807-825. doi: 10.1177/1461444815614199

This Post-Print is brought to you for free and open access by the Human Communication and Theatre at Digital Commons @ Trinity. It has been accepted for inclusion in Human Communication and Theatre Faculty Research by an authorized administrator of Digital Commons @ Trinity. For more information, please contact jcostanz@trinity.edu. 


\begin{abstract}
Relational reconnection is a prominent yet under-explored function of social network sites (SNS) that encompasses both the activation and subsequent maintenance of dormant social ties. The present investigation used two data collections (Study 1, six university sample; Study 2, national United States sample) to explore the characteristics of friends who reconnect using SNS, and attempt to predict whether reconnected relationships persisted beyond the initial reconnection. Results indicated that relational reconnection is extremely common, especially among same-sex friends and individuals who identify as heavy SNS users. Predicted outcome value emerged as the best predictor of persistence beyond initial reconnection, in addition to engaging in modality expansion, being female, and reactivating a relationship with greater perceived development preloss-of-contact.
\end{abstract}

Keywords: Relational Reconnection, Social Network Sites, Interpersonal Communication, Computer-Mediated Communication 


\section{The Relational Reconnection Function of Social Network Sites}

Scholars have begun to document the multifunctional relational nature of social network sites (SNS) beyond the functions associated with the life cycle of initiation, maintenance, and termination (see Bryant, Marmo, and Ramirez, 2011). One such function is that of relational reconnection. Ramirez and Bryant (2014) define relational reconnection as "a process that encompasses both the re-initiation of a relationship as well as its continued maintenance after initial contact" (p. 2). Individuals might lose contact with a partner for many reasons, and reconnection occurs when one partner attempts to re-establish communication.

When a reconnection attempt occurs, communicators must determine whether to reciprocate the contact, thus re-initiating the relationship, or simply allow the connection to remain dormant. If the contact is reciprocated, partners must then determine the extent to which the relationship is revitalized and incorporated into their current social network (Wilmot and Stevens, 1994). Research suggests that moving an online relationship offline is commonly regarded as an important step in its development (Gibbs et al., 2010; Ramirez et al., 2015), and the same premise might hold true for partners who reconnect on SNS. Moreover, accumulated research suggests that the number and types of channels utilized likely reflects the extent to which partners perceive positive relational qualities such as interdependence (Ledbetter and Mazer, 2014) and closeness (Caughlin and Sharabi, 2013). As such, revitalization may be reflected in the extent to which partners engage in modality expansion by extending their communication to include multiple modalities, or channels. By engaging in modality expansion, partners can increase their level of multimodality (Walther and Parks, 1996). As defined within media multiplexity theory (Haythorthwaite, 2002), multimodality, or multipexity, refers to the number of channels that are utilized to maintain a social tie. Stronger social ties typically utilize 
more communication channels than do weaker ties, which suggests that modality expansion should be important step for partners who wish to resume their newly reconnected relationship.

The present study seeks to examine relational reconnection as it occurs within the context of SNS. Such analysis will enlighten scholars to a relatively underexplored relational function of SNS, while fulfilling two main goals. First, it explores the characteristics of friends who reconnect using SNS to understand who reconnects with whom, and determine what differentiates reconnectors from non-reconnectors. Second, this study seeks to predict whether reconnected relationships persisted after initial reconnection on SNS using a set of demographic, experiential, relational, and communicative factors. The results of two studies (Study 1, six university sample; Study 2, national United States sample) are presented to address these goals.

\section{Conceptualizing Relational Reconnection}

Social network sites provide a venue for users to accomplish a multitude of relational tasks (see Bryant et al., 2011). Existing research implies that SNS are extremely common venues to maintain relationships with members of a shared offline social network, even if said relationships are of a weak tie nature (Bryant and Marmo; 2010; McEwan et al., 2014). Likewise, according to Raacke and Bonds-Raacke (2008) more than half of Facebook and MySpace users have utilized SNS to make new friends. Most SNS users are hesitant to form relationships with users they have never met in an offline capacity, and instead use the sites to interact with people they already know or share an unarticulated or latent offline social connection (Lampe et al., 2006). Similar to initiation and maintenance, reconnecting with past partners may be one manner in which SNS users position themselves to reap the social capital benefits associated with possessing a large and diverse social network (Bryant et al., 2011). 
The explosive growth and increased mobility of the Internet have greatly aided communicators in their quest to reestablish relationships from their past that would have been difficult to pursue in offline settings. As Madden and Smith (2010) note, "re-establishing connections and gathering information about people we have lost touch with is a hallmark of people search in the digital age', (part 3, para. 5). The links between relational reconnection and the Internet are, indeed, well documented. In their analysis of Pew Internet \& American Life data, Madden and Smith (2010) reported that $40 \%$ of adults under the age of 50 had been recipients of contact from a past partner with whom they previously lost touch, with 53\% of this group having used the Internet to seek information about someone from their own past. Although reconnectors might utilize many tools during the reconnection process, SNS appear to serve as a catalyst. In Madden and Smith's data, 63\% of SNS users reported conducting online searches about a past relational partner compared to only $30 \%$ of non-SNS users.

It is worth noting that partners targeted for reconnection represent once active but now dormant relationships. Social ties are said to be inactive or dormant when prior relational partners no longer possess open lines of communication, yet maintain a sense of relational history and social connection (Haythornthwaite, 2002). While some relationships are purposely terminated, most simply drift away due to lost proximity and competing time constraints (Burt, 2002). However, according to Levin, Walter, and Murnighan (2011), "as long as the option of a future reconnection continues to exist, dormant ties are not dead" (p. 923). From this perspective, relational reconnection involves activating a dormant relationship by reestablishing lines of communication and tapping back into the previous sense of relational history.

Ramirez and Bryant (2014) note that SNS provide communicators with three primary advantages that encourage the researching and activating of dormant ties during the reconnection 
process. First, SNS enable users to conduct purposive searches for specific individuals from their past who also use that site. Second, many SNS offer suggestions for potential connections via algorithms that detect similarities in the profile content of users with overlapping social ties. Third, the inherently social and public nature of SNS increase the likelihood that users will serendipitously stumble upon someone from their past while interacting on the site. Due to these collective advantages, SNS provide users with relatively unobtrusive and therefore low facethreatening opportunities to seek and gather social information about a dormant relational partner while determining whether a reconnection is desirable.

Reactivating a dormant social tie can be advantageous, as it is one way for individuals to increase the size of their active social network. Social capital scholars have long noted that most people engage in few close relationships, yet possess large social networks consisting of hundreds or even thousands of weak social ties (Granovetter, 1973). Weak ties involve low levels of intimacy, fewer and shorter interactions, reliance upon pre-organized or opportunistic means of sending CMC messages (Haythornthwaite, 2002), as well as less relational closeness and satisfaction than close relationships (Baym, Zhang, Kunkel, Ledbetter, \& Lin, 2007). Despite their superficial nature, weak ties gain utility via numbers and are associated with benefits such as increased social capital (Kavanagh, Rees, Carroll, \& Rosson, 2003) and the further expansion of their social network (Wuthnow, 1998). It is therefore easy to understand why individuals might reconnect with a dormant partner. Individuals who reconnect can then employ SNS to maintain a weak tie, which requires relatively little effort while providing numerous social benefits. Indeed, in Levin et al.'s (2011) study of business executives, reconnected partners were found to provide both efficiency and novelty of information. Consequently, SNS appear to be beneficial tools for reinvigorating dormant relationships and activating social capital. 


\section{The Present Investigation}

The present investigation examines relational reconnection as a unique and relatively unexplored function of SNS (Bryant et al., 2011). As a starting point, it is important to document the prevalence of reconnection experiences as a point of comparison with previous reports (Madden and Smith, 2010; Ramirez and Bryant, 2014).

Research Question 1: What proportion of participants report reconnecting with a friend through SNS?

Beyond noting the prevalence of relational reconnection, it is useful to examine the types of dormant relationships that individuals attempt to re-activate using SNS. Related research reports that social networks are comprised primarily of acquaintances (Parks, 2007), with the majority of them being same-sex friendships (Fehr, 1996). Although a complete review of the literature on friendships is beyond the scope of this investigation, two characteristics seem to suggest the likelihood of a preference for reconnecting with same-sex friends relative to crosssex ones. First, same-sex friendships are more populous in social networks, with both males and females communicating and spending more time, in general, with same-sex rather than cross-sex friends (see Fehr, 1996). Second, same-sex friendships, particularly among females, are typically rated as possessing greater levels of closeness and intimacy relative to cross-sex friends (e.g., McCoy et al., 1994). Taken together, the fact that the pool of same-sex friendships, relative to cross-sex ones, appears to be both larger in quantity and associated with more unique relational benefits, suggests a higher likelihood of seeking out same-sex friends from one's past.

Hypothesis 1: Reconnection with a same-sex friend will be reported more often than reconnection with a cross-sex friend. 
Another important aspect of understanding the reconnection process is exploring the factors that distinguish participants who report a reconnection experience from those who do not. Because no study to date has investigated what discriminates "reconnectors" from "nonreconnectors" it is difficult to propose a precise prediction as to which characteristics would accurately do so, however, related research on Internet relationship initiation and maintenance offers suggestions. In their study of online friendship formation, Parks and Floyd (1996) reported that the duration of membership and frequency of participation were the best predictors of who formed relationships on discussion boards. Individuals who had been members for a longer period and posted more frequently on the boards were more likely to have formed a friendship with another member. Other research suggests that having a large number of acquaintances linked to one's SNS profile increases its visibility (Tong et al., 2008), thereby making users more likely to become reconnection targets. A substantial body of literature also reports females are more likely to maintain relationships and reestablish family connections via CMC than are males (e.g., Punyanunt-Carter and Hemby, 2006). Despite being potentially informative, relational reconnection was not the focus of these studies, necessitating the following question:

Research Question 2: What factors differentiate participants who report reconnecting with friends (i.e., reconnectors) from those who do not (i.e., non-reconnectors)?

A primary influence upon the decision to reconnect with a past relational partner is likely how the nature of the pre-loss-of-contact relationship is recalled. Understanding the qualities attached to that prior relationship is important for two primary reasons. First, how individuals characterize a past relationship likely influences their decision to resurrect it. Partners who still portray their past relationship in a positive light should be more strongly motivated to revive the connection, and as noted above, benefit from the social capital that doing so may provide. 
Second, not all relationships that are reinitiated persist over time. It is likely that the qualities attached to the reconnected relationships play a central role in its continuation beyond initial contact. For example, partners who viewed their pre-loss-of-contact relationship in positive terms might be more likely to continue their relationship after reestablishing communication than partners who had a more tenuous prior relationship.

The present investigation employed multiple conceptual perspectives to assess the manner in which individuals evaluated their relationship prior to reconnecting. The first perspective involved a set of dimensions identified by Parks and Floyd (1996) in their study of friendships formed on Internet discussion boards. Drawing upon uncertainty reduction (Berger and Calabrese, 1975) and social penetration (Altman and Taylor, 1973) theories, Parks and Floyd (1996) proposed a set of characteristics which were conceptualized as descriptive of a relationship's developmental state: the degree of interdependence between partners, the breadth and depth of their communication, the extent to which partner communication exhibits code change or a specialized manner specific to the relationship, the amount of predictability/understanding between partners, their level of commitment, and the degree to which their networks converge or overlap. A second, more global means of assessing how individuals perceive their past association is to examine the extent to which the previous relationship was expected to be rewarding at that time and into the future. According to predicted outcome value (POV; Sunnafrank, 1986; 1988) theory, such an evaluation is reflective of how individuals perceive partner attitudes and behaviors towards them, as well as being predictive of the relationship's future state (Ramirez et al., 2010; Sunnafrank and Ramirez, 2004).

As previously alluded to within hypothesis 1, the nature of the pre-loss-of-contact relationship might also vary depending on whether the relationship is same-sex or cross-sex. In 
particular, same-sex friends tend to report greater intimacy and closeness than do cross-sex friends (McCoy et al., 1994). That said, previous research has focused on the current relational nature, whereas the present study is focused on understanding what the relationships were like prior to losing contact. It is uncertain whether having lost contact will affect the ways in which same-sex and cross-sex friendships recall their pre-loss-of-contact relationship. The present study therefore utilizes both POV and the relational dimensions in order to address the following: Research Question 3a: How do participants rate the pre-loss-of-contact nature of their reconnected friendships along the (a) seven developmental dimensions and (b) POV? Research Question 3b: Do same-sex and cross-sex friendships differ in their ratings of the pre-loss-of-contact nature of their reconnected friendships along the (a) seven developmental dimensions and (b) POV?

While it is important to understand the prior relationship between two reconnected partners, it is equally essential to probe the communication occurring after the initial reconnection. Questions regarding whether partners in reconnected relationships engage in modality expansion by communicating outside the SNS are of particular importance. It is well established that the Internet can facilitate the development of close relationships that transcend the boundaries of a website (Dimmick et al., 2011). In a study of Last.fm, a music oriented SNS, Baym and Ledbetter (2009) found that although shared music interests led users to interact on the site, the development of relational closeness was related to the adoption of multiple modes of communication beyond Last.fm. With respect to reconnection, this suggests that partners who perceived their prior relationship as having been close may utilize other modalities to complement or even substitute for the SNS where they reestablished contact. 
Beyond its pragmatic implications, engaging in modality expansion holds symbolic value for partners enacting relational reconnection. Exchanging personal email addresses and cell phone numbers suggests a certain level of trust that is influenced by the strength of their prior relationship, and may signal the desire to resume the connection in its previous instantiation. Individuals with prior strong ties, relative to weaker ones, should thus be more likely to expand modalities as a means of opening more interaction opportunities and reinvigorating the relationship (Miczo et al., 2011). Weak ties tend to be characterized as possessing lower levels of closeness and satisfaction (e.g., Baym et al., 2007) as well as lower communication needs than strong ties (Haythornthwaite, 2002), and engaging in modality expansion might actively strengthen the tie. Conversely, limiting interaction to the SNS following initial contact may reflect a desire to keep the connection at a superficial and channel contingent nature.

Media multiplexity theory (Haythornthwaite, 2002) suggests that stronger social ties will "adapt and expand their use of media to support the exchanges important to their tie" (p. 385). This indicates that the extent of modality expansion should be related to relational characteristics such as POV (Sunnafrank, 1986; 1988) and Parks and Floyd's (1996) developmental characteristics. However, past research refers to the strength of tie as concurrent to the number of channels utilized, whereas the present study is concerned with pre-loss-of-contact developmental characteristics. It might be assumed, based on media multiplexity theory, that individuals with greater pre-loss-of-contact relational development would engage in more modality expansion after reconnecting in an attempt to reinvigorate their previously stronger tie. That said, it is vital to remember that all reconnected relationship were left dormant, which could signal that any preloss-of-contact development no longer exists. The following research questions are thus posed: 
Research Question 4a: To what extent do friendships reconnected through SNS engage in modality expansion?

Research Question 4b: Does the extent of modality expansion relate to the pre-loss-ofcontact nature of their reconnected friendships along the (a) seven developmental dimensions and (b) POV?

Finally, a significant aspect of the relational reconnection process left to be examined concerns the reasons why reconnections might persist beyond initial SNS contact. Factors such as the relational characteristics discussed above, and the extent to which partners expand the types of modalities available for interacting should have consequences for decisions to maintain reconnections; however, their precise role is unclear. For example, Ramirez and Bryant (2014) reported that participants in reconnected relationships that persisted characterized their past associations as being more rewarding and predictable, exhibiting greater breadth of conversations, possessing greater commitment, displaying significant network overlap, and utilizing relatively more communication channels than did those that did not persist. Two caveats need to be pointed out. Although the authors detected certain correlations, their analysis was not designed to predict persistence. As a result, the relative contribution of each characteristic remains unclear. In addition, their analysis was limited to relational characteristics and did not examine other potential influences upon persistence, such as demographic data (e.g., sex, age), or Internet and SNS experience. The present study expands on Ramirez and Bryant's (2014) analysis by addressing both of these shortcomings. The final research question asks:

Research Question 5: Which factors predict whether a reconnected relationship will persist beyond the initial reconnection?

\section{Method}


The present investigation reports on two empirical studies examining how individuals utilize SNS to reconnect with relational partners from their past. Study 1 employed a collegeaged sample to investigate the relational reconnection process, whereas study 2 replicated the initial study using a national sample of SNS users.

\section{Study 1}

Participants. Data for study 1 were collected from 845 participants (313 males, 532 females) recruited from communication classes at six universities and colleges of varying size and geographic location. The average age of the final sample was 21.27 years $(S D=3.14)$. The majority of the sample self-identified as White/Caucasian $(78.3 \%, n=662)$, with AfricanAmerican $(8.0 \%, n=68)$, Hispanic/Latino $(5.1 \%, n=43)$, and Asian-American $(4.7 \%, n=40)$

also represented; thirty-two (3.8\%) participants either identified themselves as "other" or did not provide this information. When possible, extra class credit was offered in exchange for participation. Preliminary analyses indicated that the experience of relational reconnection did not differ among campuses $(p>.22)$, suggesting the institution was not a factor in reconnection opportunities. Consequently, data were collapsed across locations for the analyses reported.

Procedure and measures. Participants were provided a URL address to access a human subjects consent form, and three section online questionnaire. After providing consent, participants were directed to the first section of the questionnaire, which included demographic questions such as age, sex, ethnicity, and level of experience using the Internet and SNS.

The second section included items asking about participants' experience with relational reconnection and began with the following description:

"Individuals use social network sites for a variety of reasons. For instance, although these sites are used to initiate new relationships and maintain existing ones, some have reported 
re-connecting with family, friends, and others with whom they had lost contact with at some point. The following questions focus on the use of social network sites for this reason."

Participants were then asked to indicate whether or not they had reconnected with someone from their past using an SNS during the previous year. Those answering affirmatively were directed to remainder of the questionnaire, whereas the other participants were directed to an alternative study site. Although the focus of the present study is friendships, respondents were asked to report on any reconnection in order to minimize potential demand characteristics that may be associated with participating in the study for extra credit. Responses about relationships other than friendships were removed from all analysis beyond RQ1, as were responses from participants who had not reconnected on SNS in the previous year.

On the following page, participants were asked a series of questions about the reconnection experience, their partner, and the reasons for initially losing contact. Those indicating they had reconnected with more than one person within the last year were instructed to report on their most recent instance. Participants were asked to report the pre-loss-of-contact relational characteristics along Parks and Floyd's (1996) seven relationship development measures as well as Sunnafrank's (1988) measure of predicted outcome value (POV) forecast (see Table 1 for reliability estimates). The Parks and Floyd measures employ a 5-point scale, whereas Sunnafrank's instrument utilizes a 6-point scale. Higher scores represent greater levels on each measure. In order to measure the level of modality expansion, participants were next presented a set of communication channels (personal email, instant messaging, text messaging, telephone, and face-to-face) and asked to indicate whether they had used each with their partner since the initial reconnection. The final section focused on the current state of the relationship, 
and measured persistence with a nominal (yes/no) question that asked whether they were still in contact with their reconnected partner.

\section{Study 2}

Participants. Study 2 replicated the initial study with the exception of utilizing data collected from a national sample of SNS users. Participants were recruited through a market research firm that maintains research panels. Initial recruitment occurred via email contact from the firm announcing the study, its focus, and participation requirements. Potential participants received a follow-up email approximately 10 days after the initial contact reminding them of the study's availability. All participants, irrespective of whether they qualified for the full study, received a nominal financial incentive from the market research firm in order to minimize demand characteristics in the data collection. The final sample was comprised of 346 participants (156 males, 190 females) with a mean age of 31.89 years $(S D=7.58)$. Participants self-identified primarily as White/Caucasian $(79.5 \%, n=275)$, although African-American $(9.2 \%, n=32)$, Hispanic/Latino $(4.3 \%, n=15)$, and Asian-American $(4.3 \%, n=15)$ were also represented; nine (2.6\%) participants either identified themselves as "other" or did not provide this information.

Procedure and measures. Study 2 mirrored the initial study in its procedures and materials with the consent form modified per Institutional Review Board requirements pertaining to the use of a market research firm (i.e., statement of responsibility). Minor adjustments were also made in the wording of the instructions where necessary due to the use of a non-college sample; the materials were otherwise identical.

\section{Results}

\section{Research Question 1}


Research question 1 asked what proportion of SNS users would report reconnecting with a friend from their past. Relational reconnection was quite common in study 1 . A total of 622 (73.6\%) of the 845 SNS users who participated in the study reported at least one reconnection experience during the course of the last year; $56.3 \%$ occurred within the last 6 months. Of those reporting at least one experience, friends comprised $508(81.7 \%)$ of the reconnections. Approximately $40.25 \%(N=225)$ reported initiating the contact, whereas $59.75 \%(N=334)$ reported being the recipient of such a contact. Participants reported being out of touch with their partners for an average of 49.34 months $(S D=39.43)$ at the time of reconnection, with a range from 12 to 192 months.

The national sample employed in study 2 reported a prevalence rate slightly higher than that of the first. A total of $346(84.59 \%)$ of the 409 SNS profile owners in the sample reported reconnecting with at least one person during the last year, with most occurring within the last 6 months (68.2\%). Of these reconnections, friendships comprised 323 (93.35\%) of those reported. In line with study 1 , participants reported being the recipients $(N=187,57.89 \%)$ more often than the initiators of the contact $(N=136,42.1 \%)$. Participants reported being out of touch for a longer time period of 98.74 months $(S D=43.18)$, with a range from 18 to 256 months.

\section{Hypothesis 1}

Hypothesis 1 predicted that reconnection with a same-sex friend would be reported more often than with a cross-sex one, and this was supported by both studies. In study 1 , reconnection with same-sex friends $(N=323,63.6 \%)$ was reported proportionately more often than that with cross-sex friends $(N=185,36.4 \%), \chi^{2}=36.94, p<.001$. Likewise, participants in study 2 reported reconnecting with same-sex friends $(N=225,69.7 \%)$ proportionately more often than with cross-sex friends $(N=98,30.3 \%), \chi^{2}=49.94, p<.001$. 
Friendships and SNS Reconnection 16

\section{Research Question 2}

Research question 2 asked which factors distinguish participants who reported reconnecting with a friend from those who did not. The analysis associated with study 1 revealed significant differences on several factors related to SNS use that differentiated reconnectors from non-reconnectors. Reconnectors reported having profiles across relatively more SNS sites $(M=$ $1.59, S D=0.62$, vs. $M=1.34, S D=0.52), t(843)=5.38$, two-tailed $p<.001$, and visited their sites more often $(M=5.84$ days/week, $S D=1.75$, vs. $M=5.21$ days/week, $S D=2.47, t(843)=$ 4.11, two-tailed $p<.05$, than non-reconnectors. Reconnectors, however, did not differ from their counterparts in terms of the number of "friends" linked to their profiles $(p>.20)$, or the length of time having had a profile $(p>.27)$. Biological sex also appears not to have a significant effect on reconnection, with men and women equally likely to report the experience $(p>.40)$.

A similar pattern emerged in the national sample used in study 2 . Reconnectors reported owning profiles across relatively more SNS $(M=1.66, S D=0.57$, vs. $M=1.33, S D=0.57)$, $t(407)=4.17$, two-tailed $p<.001$, and visited those sites more often $(M=5.73$ days/week, $S D=$ 1.89 , vs. $M=4.86$ days/week, $S D=2.52), t(407)=3.21$, two-tailed $p<.001$, than non-

reconnectors. The groups, however, were not significantly different with respect to the number of "friends" reported $(p>.26)$, or the length of time owning a profile $(p>.29)$. Sex differences also failed to surface in terms of reporting a reconnection experience $(p>.36)$.

\section{Research Question 3}

Research question 3a sought to understand how reconnectors perceived their relationships prior to having lost contact, and $3 \mathrm{~b}$ asked whether same-sex and cross-sex friendships differ in this regard. Reconnectors were asked to record their perceptions of the relationship prior to losing contact (i.e., what the relationship was like) on Parks and Floyd's (1996) developmental 
dimensions as well as Sunnafrank’s (1986) POV measure. Table 2 reports descriptive information for each dimension overall and by friendship type. The table also reports the results of significance tests assessing: (a) the overall level of pre-loss-of-contact relational development on each dimension relative to the mid-point of their respective measure; as well as (b) the relative level of pre-loss-of-contact development reported by same-sex and cross-sex friends.

With respect to the overall level of development, participants in study 1 rated their friendships significantly above the scale mid-point on five of the dimensions (breadth, depth, predictability/understanding, commitment, network convergence), with only one (code change) significantly below it. The comparisons between same-sex and cross-sex friendships yielded significant differences on five of the dimensions. An initial multivariate analysis of variance (MANOVA) conducted on the composite of the dimensions was significant, however the effects were not consistent across each outcome (see Table 2). Assessment of the univariate analyses of variance (ANOVA) tests indicated same-sex friendships were rated as having greater interdependence, breadth, personalized communication/code change, predictability/understanding, and commitment relative to cross-sex ones. Analyses of POV, depth, and network convergence failed to achieve statistical significance.

The overall level of development reported by participants in study 2 was significantly different than the scale mid-point on each dimension; once more, only the level of code change was reported significantly below its scale mid-point, with the rest falling above. The MANOVA conducted on the composite of the dimensions produced a significant multivariate effect, but once more the effects were not consistent across outcomes. Examination of the accompanying ANOVAs showed participants rated their same-sex friendships significantly higher on five 
dimensions (interdependence, depth, code change, predictability/understanding, commitment). Analyses of POV, breadth, and network convergence failed to achieve statistical significance.

\section{Research Question 4}

Research question 4a asked whether partners who reconnect through SNS engage in modality expansion. The results associated with study 1 suggest that the majority of reconnectors do, indeed, expand the number of modalities they use to communicate with reconnected partners. The mean number of channels reported was $1.94(S D=1.52)$, and 47.4\% $(N=301)$ reported using three or more channels to communicate with their reconnected partner. In terms of specific channels, a substantial percentage reported using personal email $(N=210,33.8 \%)$, instant messaging $(N=250,40.2 \%)$, text messaging $(N=232,37.3 \%)$, and phone calls $(N=260$, 41.8\%). Almost one-third reported meeting their partner in person $(N=193,31.6 \%)$ after the initial reconnection. Conversely, slightly more than 1 in 5 respondents $(N=128,20.6 \%)$ reported limiting their communication to the SNS itself.

Reconnectors in study 2 also frequently expanded the number of modalities used to communicate with their partner. They reported an average of $1.79(S D=1.68)$ channels, with $35 \%(N=112)$ using three or more channels to communicate with their partner. The sample reported using numerous modalities including personal email $(N=119,36.8 \%)$, instant messaging $(N=87,26.9 \%)$, text messaging $(N=120,37.2 \%)$, and phone calls $(N=145,44.9 \%)$. Approximately 1 in $3(N=102,31.6 \%)$ participants reported meeting their partner in person following initial reconnection. Less than 1 in $4(N=73,22.6 \%)$ reported not expanding their relationship beyond the SNS.

Research question $4 \mathrm{~b}$ asked whether modality expansion is related to pre-loss-of-contact relational development characteristics. To test this, Pearson correlations were computed for the 
number of channels used and each development dimension. Study 1 participant reports of the total number of channels used were moderately associated with all of the dimensions: POV, $r=$ $.32, p<.001$; interdependence, $r=.35, p<.001$; breadth, $r=.42, p<.001$; depth, $r=.45, p<$ .001 ; code change, $r=.28, p<.001$; predictability/understanding, $r=.29, p<.001$; commitment, $r=.26, p<.001$. Participants in study 2 reports of the total number of channels used were also significantly associated with the dimensions: $\mathrm{POV}, r=.34, p<.001$; interdependence, $r=.31, p$ $<.001$; breadth, $r=.36, p<.001$; depth, $r=.41, p<.001$; code change, $r=.22, p<.01$; predictability/understanding, $r=.26, p<.001$; commitment, $r=.29, p<.001$.

\section{Research Question 5}

Research question 5 asked which factors differentiate reconnections that persisted from those that did not. This research question was investigated through the use of logistic regression analysis. In order to identify the combination of factors that would be predictive of persistence, participant reports of whether they were still in contact with their friend $(0=$ no, $1=$ yes $)$ at the time of the study were regressed on the following set of predictors: demographic characteristics (respondent and partner sex, age), experience using the Internet and SNS, type of friendship, the seven developmental dimensions, POV, and level of modality expansion. The likelihood ratio test (-2 log likelihood) in logistic regression compares model deviances to assess whether the model with the predictors provides a better fit for the data than the one without any predictors. The test also yields a one-degree of-freedom $\chi^{2}$ statistic that tests the null hypothesis that adding the predictors does not explain variance beyond that of the baseline predictor model.

Table 3 reports the results of the analyses for both studies; all statistics reported are for the final models. For study 1, the analysis identified five characteristics that differentiated relationships that persisted from those that did not. Respondent sex, POV, depth, 
predictability/understanding, and the total number of channels used positively predicted persistence. The only other predictors to approach significance were interdependence $(p=.09)$ and breadth $(p=.10)$. The overall findings for study 2 are fairly consistent with those of the initial study. Once more, respondent sex, POV, predictability/understanding, and total number of channels used emerged as significant predictors of persistence. In addition, the analysis also identified interdependence as a significant predictor. However, unlike with study 1 the relational dimension of depth $(p=.09)$ failed to achieve statistical significance. Partner sex $(p=.10)$ was the only other predictor to approach significance.

\section{Discussion}

The present investigation represents the first in-depth examination of the relational reconnection function of SNS, a function that encompasses both the activation of dormant ties as well as their maintenance beyond initial contact. Building upon the research of Ramirez and Bryant (2014) and Madden and Smith (2010), the present research advances our understanding of the reconnection process by addressing two important goals. First, the present investigation documented the characteristics of reconnectors and their relationships. Second, it identified a set of characteristics that could predict the persistence of reconnected relationships. The overall results provide a rather robust assessment of the nature of relational reconnection and make several contributions to the scholarly literature.

\section{Characteristics of Reconnectors and their Friendships}

Four of the research questions and one hypothesis were aimed at better understanding the characteristics of reconnectors and their reconnected friendships. The findings across studies reveal that reconnection is incredibly common, with approximately three-fourths of participants reporting having reconnected with someone from their past. More than half occurred within the 
previous six months, with friends being the most common form. Specifically, the participants were more likely to report being the recipient of their most recent reconnection, as opposed to the initiator of it. These findings complement Pew Internet and American Life data indicating that $63 \%$ of SNS users have conduced online searches about a past partner (Madden and Smith, 2010), while reflecting the continued growth of SNS. The present investigation, however, took this concept a step further by identifying whether the initial contact actually translated to a reconnected relationship.

The question of what characteristics distinguish those who report engaging in reconnection via SNS was also of interest. Previous research suggests factors such as duration of group membership and frequency of participation (Parks and Floyd, 1996) as well as visibility on SNS (e.g., Tong et al., 2008) might affect the likelihood of experiencing reconnection. The findings in these respects were largely consistent across the two studies: reconnectors reported having more SNS profiles and using said profile more frequently than non-reconnectors; the groups did not differ in regard to other characteristics (number of friends, length of time since joining SNS, or biological sex). As a whole, these results reveal a mostly intuitive picture in which individuals who use SNS more intensely are more likely to reconnect as a result of their relatively heavier use.

The present research also begins to paint a portrait of the nature of reconnected relationships. The reconnected relationships participants reported on were moderately welldeveloped prior to loss-of-contact. Comparisons of the reported levels of development and the mid-point of each measure showed the rejuvenated relationships had achieved pre-loss-ofcontact development that was significantly, albeit slightly, above the mean for most measures, which probably served as part of the impetus for connecting once more. Moreover, SNS users 
are significantly more likely to report having reconnected with a same-sex friend as opposed to a cross-sex one. These results align with research indicating that same-sex friends spend more time together (Fehr, 1996) and possess more relational closeness (e.g., McCoy et al., 1994) than crosssex friends, which might provide increased incentives for reconnection. Additionally, these relationships appear to be characterized by a fairly consistent set of qualities. As expected per prior research (e.g., McCoy et al., 1994), same-sex friendships were reported as being of greater relational development than were cross-sex friendships before having lost contact. In the present investigation, this emerged in the form of greater interdependence, depth, predictability/understanding, and commitment. Thus, it appears SNS users are most likely to reconnect with same-sex friends, particularly those with whom they previously had a fairly yet not overly developed association before it became dormant.

Once reconnected, many partners appear to engage in modality expansion, and the preloss-of-contact relational development characteristics were consistently associated with the number of channels used after reconnecting. Modality expansion encompasses the extent to which relational partners developed media multiplexity by expanding their communication to include various channels beyond the SNS. Recent research connects relational qualities such as greater interdependence (Ledbetter and Mazer, 2014) and closeness (Caughlin and Sharabi, 2013) to the diversity of channels partners employ in their relationship. The present investigation shows that many reconnectors engaged in some degree of modality expansion, with more than one-third of each sample reporting using 3 or more channels with their partner, and approximately one-third having also met in a FtF setting. In contrast, approximately one-fifth of reconnectors did not engage in modality expansion and limited any subsequent communication to the SNS itself. For these individuals, reconnecting might serve little purpose beyond bringing 
their dormant friendship back into their realm of social awareness. Nonetheless, consistent with the notion of media multiplexity (Haythornthwaite, 2002), the reconnectors who did expand their communication should be better suited to redevelop the greater levels of closeness that once characterized their relationship as a function of their increased media repertoire.

\section{Predicting Relational Persistence Following Reconnection}

The second primary goal of the present investigation was to identify which factors predict relational persistence after initial SNS reconnection. Ramirez and Bryant (2014) examined a similar question, yet focused only on the relational characteristics that might be related to persistence, thus not accounting for other potentially important predictors, such as demographic data (e.g., sex, age), Internet and SNS experience, and who initiated the reconnection. Likewise, the authors only reported individual correlations without attempting to predict relational persistence within a model that could identify the relative importance of each factor.

The present analyses revealed a fairly consistent set of factors that account for relationship persistence. Respondent sex, the total number of channels utilized, and three preloss-of-contact relational characteristics collectively predicted relational persistence in study 1. Similar results emerged in study 2 , with one relational characteristic dropping out of the model (depth), and a different characteristic becoming significant (interdependence). Irrespective of this one difference, a fairly clear pattern emerged across the two studies: being female, engaging in modality expansion, and reactivating a relationship attributed greater pre-loss of contact development significantly increases the likelihood the relationship persists. Similar to Ramirez and Bryant (2014), POV emerged as the best pre-loss of contact predictor in study 1, and the second best predictor in study 2 , which supports the notion that a relationship is more likely to persist when the partner feels that doing so will continue to produce positive outcomes. Indeed, a 
positive pre-loss-of-contact POV, coupled with the slightly above the mean level of relational development seen in research question 3a, suggests that the partners simply fell out of touch for some benign reason, and thus welcomed the renewed contact. The number of channels used (i.e., degree of modality expansion) emerged as the second strongest predictor in study 1 , and was the strongest predictor in study 2 , which again speaks to the relevance of media multiplexity (Haythornthwaite, 2002).

One potential explanation for the collective results regarding persistence is that individuals choose to expand their communication when they renew a previously well-developed relationship. In other words, the strength of tie drives the subsequent modality expansion and relational persistence. This interpretation aligns with Haythornthwaite's (2002) observation that media multiplexity often reflects that stronger ties possess closer relationships with greater communicative needs. This explanation is particularly likely due to the present study's finding that pre-loss-of-contact relationship characteristics predicted both the degree of modality expansion and also persistence. A second and related explanation for this finding is that modality expansion can actually help to strengthen weak ties (Baym et al., 2007). Indeed, latent tie theory (Haythornthwaite, 2005) claims that weak ties are more channel contingent, and thus more heavily affected by the addition or subtraction of a channel than are strong ties. Although reconnectors are drawing upon a previous social connection, they are still attempting to reinvigorate it from a completely dormant state. As such, engaging in modality expansion and developing media multiplexity can be an important step for newly reconnected partners who wish to go from a dormant tie to a weak tie, and perhaps even develop a stronger tie that persists beyond the initial contact.

\section{Limitations and Future Directions}


The present investigation, due to its limitations, offers several opportunities for future research. One limitation is that the investigation provides insight into the reconnection of only friendships on SNS. Although other relationships were reported in the initial data collections, their numbers were insufficient to allow for further analyses. Clearly, future research should attempt to gather data from a broader array of relationship types. Another limitation is that the present study provides only a snapshot of the reconnection process. Equally important as whether reconnections had persisted at the time of the data collections is whether they persist over an even longer extended period. Because the majority of reconnections reported occurred within the previous six months, it would be worthwhile to track their persistence more longitudinally to assess their continued viability. Finally, although the investigation focused on how SNS facilitate reconnection, it did not concentrate on design aspects that may help to fulfill this function beyond simply offering suggestions for potential connections. That is, it may be that certain sites are designed in ways that make reconnection more possible than do others. Future research should attempt to identify such features and assess their impact relative to other factors in facilitating reconnection. As is, however, the present investigation offers an important conceptualization and initial investigation into the relational reconnection function of SNS, and can serve as a springboard for additional research on the topic. 


\section{References}

Altman I and Taylor D (1973. Social Penetration: The Development of Interpersonal Relationships. New York: Holt.

Baym NK and Ledbetter A (2009) Tunes that bind?: Predicting friendship strength in a musicbased social network. Information, Communication \& Society 12: 408-427.

Baym NK, Zhang YB, Kunkel A, Ledbetter A and Lin M (2007) Relational quality and media use in interpersonal relationships. New Media \& Society 9: 735-752.

Berger CR and Calabrese RJ (1975) Some exploration in initial interaction and beyond: Toward a developmental theory of communication. Human Communication Research 1: 99-112.

Bryant EM and Marmo J (2010) Relational maintenance strategies on Facebook. The Kentucky Journal of Communication. Available at: http://kycommunication.com/jenniferpdf/Bryant.pdf

Bryant EM, Marmo J, and Ramirez Jr. A (2011). A functional approach to social networking sites. In: Wright KB and Webb LM (eds) Computer-Mediated Communication in Personal Relationships. New York: Peter Lang, pp.3-20.

Burt R (2002) Bridge decay. Social Networks 24: 333-363.

Caughlin JP and Sharabi LL (2013) A communicative interdependence perspective of close relationships: The connections between mediated and unmediated interactions matter. Journal of Communication 63: 873-893.

Dimmick J, Feaster J and Ramirez Jr. A (2011) The niches of interpersonal media: Relationships in time and space. New Media \& Society 13: 1265-1282.

Fehr B (1996) Friendships Processes. Thousand Oaks, CA: Sage. 
Gibbs JL, Ellison NB and Heino RD (2006) Self-presentation in online personals: The role of anticipated future interaction, self-disclosure, and perceived success in Internet dating. Communication Research 33: 152-177.

Granovetter M (1973) The strength of weak ties. American Journal of Sociology 78: 1360-1380. Haythornthwaite C (2001) Exploring multiplexity: Social network structures in a computersupported distance learning class. The Information Society 17: 211-226.

Haythornthwaite C (2002) Strong, weak and latent ties and the impact of new media. The Information Society 18: 385 - 401.

Haythornthwaite C (2005) Social networks and Internet connectivity effects. Information, Communication \& Society 8: 125-147.

Kavanagh A, Rees DD, Carroll JM and Rosson MB (2003) Weak ties in networked communities. In: Huysman M, Wenger E and Wulf V (eds) Communities and Technologies. Kluwer; Dordrecht, pp.265-286

Lampe C, Ellison N and Steinfield C (2006) A face(book) in the crowd: social searching vs. social browsing. In: Proceedings of CSCW, New York: ACM Press, pp.167-170.

Ledbetter AM and Mazur JP (2014) Do online communication attitudes mitigate the association between Facebook use and relational interdependence? An extension of media multiplexity theory. New Media \& Society 16: 806-822.

Levin D, Walter J and Murnighan J (2011) Dormant ties: The value of reconnecting. Organization Science 22: 923-939.

Madden M and Smith A (2010) Reputation management and social media. Report for Pew Research Center. Washington, DC: Retrieved from: http://pewinternet.org/Reports/2010/Reputation-Management 
McCoy JK, Brody GH and Stoneman Z (1994) A longitudinal analysis of sibling relationships as mediators of the link between family processes and youths' best friendships. Family Relations 43: 400-408.

Miczo N, Mariani T and Donahue C (2011) The strength of strong ties: Media multiplexity, communication motives, and maintenance of geographically close friends. Communication Research Reports 24: 12-24.

McEwan B, Fletcher J, Eden J and Sumner EM (2014) Development and validation of a Facebook relational maintenance scale. Communication Methods and Measures 8: 244263.

Parks MR (2007) Personal Relationships and Personal Networks. Mahwah, NJ: Lawrence Erlbaum.

Parks MR and Floyd K (1996) Making friends in cyberspace. Journal of Communication 46: 8097.

Punyanunt-Carter NM and Hemby CO (2006) College students' gender differences regarding email. College Student Journal 40: 651-653.

Raacke J and Bonds-Raacke J (2008) MySpace and Facebook: Applying the uses and gratifications theory to exploring friend-networking sites. CyberPsychology and Behavior 11: $169-174$.

Ramirez Jr. A and Bryant EM (2014). Relational reconnection on social network sites: An examination of relationship persistence and modality switching. Communication Reports 27: $1-12$. 
Ramirez Jr. A, Sumner EM, Fleuriet C and Cole M (2015) When online dating partners meet offline: The effect of modality switching on relational communication between online daters. Journal of Computer-Mediated Communication 20: 99-115.

Ramirez Jr. A, Sunnafrank M and Goei R (2010) Predicted outcome value theory in ongoing relationships. Communication Monographs 1: 27-50.

Sunnafrank M (1986) Predicted outcome value during initial interactions: A reformulation of uncertainty reduction theory. Human Communication Research 13: 3-33.

Sunnafrank M (1988) Predicted outcome value in initial conversations. Communication Research Reports 5: 169-172.

Sunnafrank M and Ramirez Jr. A (2004) At first sight: Persistent relational effects of getacquainted conversations. Journal of Social and Personal Relationships 21: 361-379.

Tong ST, Van Der Heide B, Langwell L and Walther JB (2008) Too much of a good thing? The relationship between number of friends and interpersonal impressions on Facebook. Journal of Computer-Mediated Communication 13: 531-549.

Walther JB, \& Parks MR (2002) Cues filtered out, cues filtered in: Computer-mediated communication and relationships. In Knapp ML and Daly JA (Eds) Handbook of interpersonal communication $\left(3^{\text {rd }}\right.$ ed) Thousand Oaks, CA: Sage, pp.529-563

Wilmot WW and Stevens DC (1994) Relationship rejuvenation: Arresting decline in personal relationships. In: Conville D (ed) Uses of Structure in Communication Studies. Westport, CT: Praeger, pp.103-124

Wuthnow R (1998) Loose connections: Joining together in America's fragmented communities. Cambridge, MA: Harvard University Press. 
Table 1.

Reliability coefficients for developmental dimensions.

\section{Coefficients}

Developmental dimension

Study $1 \quad$ Study 2

POV

.93

.90

Interdependence

.87

.88

Breadth

.75

.80

Depth

.82

.84

Code change

.85

.86

Predictability/understanding

$\begin{array}{ll}.85 & .88\end{array}$

Commitment

.83

.83

Network convergence

.83

.86 
Table 2.

Descriptives and significance tests for developmental dimensions overall and by friendship type.

\begin{tabular}{|c|c|c|c|c|c|c|}
\hline \multirow[b]{3}{*}{ Dimension } & \multicolumn{3}{|c|}{ Study $1(N=508)$} & \multicolumn{3}{|c|}{ Study $2(N=323)$} \\
\hline & \multirow[b]{2}{*}{ Overall } & \multicolumn{2}{|c|}{ Friendship type } & \multirow[b]{2}{*}{ Overall } & \multicolumn{2}{|c|}{ Friendship type } \\
\hline & & Same-sex & Cross-sex & & Same-sex & Cross-sex \\
\hline POV & $3.54(.66)$ & $3.55(.58)$ & $3.53(.75)$ & $3.46(.67)^{* *}$ & $3.47(.65)$ & $3.45(.72)$ \\
\hline Interdependence & $2.95(.85)$ & $3.04(.81)_{\mathrm{a}}$ & $2.81(.88)_{\mathrm{a}}$ & $3.55(.84)^{* *}$ & $3.68(.83)_{\mathrm{f}}$ & $3.26(.86)_{\mathrm{f}}$ \\
\hline Breadth & $3.38(.99)^{* *}$ & $3.47(.96)_{b}$ & $3.26(1.03)_{b}$ & $3.89(.97)^{* *}$ & $3.83(.94)$ & $4.02(1.03)$ \\
\hline Depth & $3.21(.93)^{* *}$ & $3.25(.89)$ & $3.16(.98)$ & $3.66(.79)^{* *}$ & $3.73(.76)_{\mathrm{g}}$ & $3.49(.87)_{\mathrm{g}}$ \\
\hline Code change & $2.68(1.03)^{* *}$ & $2.78(.97)_{\mathrm{c}}$ & $2.57(1.09)_{\mathrm{c}}$ & $2.63(.97)^{* *}$ & $2.74(.96)_{\mathrm{h}}$ & $2.39(1.00)_{\mathrm{h}}$ \\
\hline Pred./Underst. & $3.50(.88)^{* *}$ & $3.61(.77)_{\mathrm{d}}$ & $3.37(.99) \mathrm{d}$ & $4.26(.87)^{* *}$ & $4.34(.82)_{\mathrm{i}}$ & $4.09(.97)_{\mathrm{i}}$ \\
\hline Commitment & $3.08(.89)^{*}$ & $3.20(.82)_{\mathrm{e}}$ & $2.93(.96)_{\mathrm{e}}$ & $3.86(.85)^{* *}$ & $3.97(.80)_{\mathrm{j}}$ & $3.61(.97)_{\mathrm{j}}$ \\
\hline Network converg. & $3.43(.83)^{* *}$ & $3.41(.79)$ & $3.44(.86)$ & $3.74(.85)^{* *}$ & $3.76(.83)$ & $3.68(.89)$ \\
\hline
\end{tabular}

Notes: All variables used a 5-point scale with the exception of POV which employed a 6-point scale. Significance tests for the overall means reflect comparisons with the respective scale mid-point. Means for friendship types accompanied by the same subscript indicate significant differences at the $p<.05$ level or less. All results reported are based on two-tailed tests. $* p<.05 ; * * p<.01$.

Multivariate analyses of variance (MANOVA) results: Study 1: Bartlett's test of sphericity: $\chi^{2}(128)=143.91, p<.001$, average $r=$ $.44, F(1,499)=34.42, p<.001$, Wilks' $\Lambda=.81$; Study 2: Bartlett's test of sphericity: $\chi^{2}(87)=130.69, p<.001$, average $r=.38, F(1$, $314)=25.19, p<.001$, Wilks' $\Lambda=.80$. 
Table 3.

Results for the final logistic regression models predicting persistence of reconnected friendships.

\begin{tabular}{|c|c|c|c|c|}
\hline \multirow[b]{2}{*}{ Predictor } & \multicolumn{2}{|c|}{ Study $1(N=508)$} & \multicolumn{2}{|c|}{ Study $2(N=323)$} \\
\hline & $\mathrm{B}$ & S.E. & $\mathrm{B}$ & S.E. \\
\hline Respondent sex & .522 & .265 & .637 & .274 \\
\hline POV & .901 & .243 & .931 & .216 \\
\hline Depth & .512 & .245 & - & -- \\
\hline Predictability/understanding & .530 & .249 & .681 & .219 \\
\hline Total number of channels & .755 & .101 & .746 & .099 \\
\hline Interdependence & -- & - & .539 & .223 \\
\hline Constant & .703 & .381 & .998 & .580 \\
\hline -2 Log Likelihood & -16.1 & & -10.91 & \\
\hline Model $\chi^{2}$ & 37.99 & & 49.81 & \\
\hline Pseudo $\mathrm{R}^{2}$ & .39 & & .57 & \\
\hline
\end{tabular}

Notes: For study 1, 92\% of the cases were classified correctly. For study 2, 94\% were classified correctly. 